\title{
Accelerated Frames and Galactic Rotation Curves
}

\author{
S. C. Ulhoa ${ }^{1, *}$ and F. L. Carneiro ${ }^{1,+}$ \\ ${ }^{1}$ Instituto de Física, Universidade de Brasília, 70910-900, Brasília, DF, Brazil
}

\begin{abstract}
In this article the galactic rotation curve is analyzed as an effect of an accelerated reference frame. This phenomenon is the strongest evidence for the so called dark matter. We show that a non-inertial reference frame could explain the experimental data. We also show that general relativity is not enough to complete explain that which encouraged alternatives paths such as the MOND approach. Considering the effect of dark matter as a realization of accelerated reference frames is a simple but powerful hypothesis.

Keywords: Accelerated frames; Galactic rotation curve; Dark matter.
\end{abstract}

${ }^{*}$ Electronic address: sc.ulhoa@gmail.com

${ }^{\dagger}$ Electronic address: fernandolessa45@gmail.com 


\section{INTRODUCTION}

Since the dawn of modern Astrophysics the idea of dark matter imbibed experimental data. The first one to use such a term was Zwicky [1], he noted an unexpected behavior while studying the redshift of galaxies in the Coma cluster. Then more evidences were gathered along the years, for instance, in the pattern of Cosmic Microwave Background (CMB) and in the large structure formation, dark matter should play an important hole. Perhaps the most famous evidence is the galactic rotation curves [2]. In such curves the rotation velocity of stars versus their distance to the galaxy center is not well understood by current theories. The very nature of dark matter remains obscure, attempts to direct detect it, up to now, were unsuccessful $[3,4]$. Thus candidate after candidate was discarded due to some experimental constraint.

Milgrom proposed a modification in the Newtonian dynamics in order to explain the galaxy rotation curves [5]. Such a proposition gave rise to the so called MOND approach which reasonable fits experimental data [6,7]. Inteady of assuming the existence of a non-luminous matter, MOND suggests the introduction of a new fundamental constant that modifies Newtonian gravity up to a certain limit. On the other hand such Newtonian limit is not suitable to describe neither a galaxy nor a large structure dynamics. Thus this MOND method is more likely to be an effect of general relativity. Following this idea Bičák showed that general relativity can reproduce the behavior of the galactic rotation curve, modeling such structure as a rotating flat disc [8]. Then another attempts have been made trying to obtain what is observed with enhanced models [9].

Although alternative explanations to dark matter exists, the search for such an exotic matter continues. Maybe, after so many years without a positive result in its detection, it is time to expand alternative explanations for the phenomena. In this article we present one of such alternatives. Possibly we are observing the features of a non-inertial reference frame. Accelerated reference frames seems to be absolute, for instance a charge accelerated with relation to a inertial reference frame irradiates as the laws of Electrodynamics predict. However an entire different behavior occurs when an accelerated reference frame analyzes an static charge. In fact there is no symmetry between these two situations as one should expect if the relativity principle is valid for acceleration [10]. In [11] dark energy was understood as an effect of accelerated reference frame. Maybe such an effect cannot alone explain all evidences for dark matter, but certainly can not be ignored.

This article is divided as follows. In section II we present a model for a galaxy, considering general relativity. In section III we introduce the acceleration tensor and use it to obtain the galactic rotation curve in section IV. In the last section we make our final remarks. We use geometric 
unities with $G=c=1$, unless otherwise stated.

\section{A SIMPLE GALAXY MODEL}

In this section we propose a simple model to describe a galaxy. It consists in a rotating cylinder whose metric is $[12,13]$

$$
d s^{2}=-e^{2 \psi(\rho, z)} d t^{2}+e^{-2 \psi(\rho, z)}\left[e^{2 \gamma(\rho, z)}\left(d \rho^{2}+d z^{2}\right)+\rho^{2} d \phi^{2}\right] .
$$

Most of the galaxy mass is concentrated in a central region while stars describe a close motion around it. Thus the vacuum solution of Einstein equation is enough to predict the galactic rotation curve since the mass of the star is much smaller than the central mass distribution.

The non-vanishing components of the Ricci tensor, constructed out of Christoffel symbols ${ }^{0} \Gamma_{\lambda \mu}^{\sigma}$ ' are

$$
\begin{aligned}
& R_{00}=\frac{1}{\rho}\left(\rho \partial_{z} \partial_{z} \psi+\partial_{\rho} \psi+\rho \partial_{\rho} \partial_{\rho} \psi\right) e^{4 \psi-2 \gamma}, \\
& R_{11}=\frac{\partial_{\rho} \gamma-\rho \partial_{\rho} \partial_{\rho} \gamma-2 \rho\left(\partial_{\rho} \psi\right)^{2}+\partial_{\rho} \psi+\rho \partial_{\rho} \partial_{\rho} \psi}{\rho}-\partial_{z} \partial_{z} \gamma+\partial_{z} \partial_{z} \psi, \\
& R_{22}=\rho e^{-2 \gamma}\left(\rho \partial_{z} \partial_{z} \psi+\partial_{\rho} \psi+\rho \partial_{\rho} \partial_{\rho} \psi\right), \\
& R_{13}=\frac{\partial_{z} \gamma}{\rho}-2 \partial_{z} \psi \partial_{\rho} \psi, \\
& R_{33}=-\partial_{z} \partial_{z} \gamma-\frac{\partial_{\rho} \gamma}{\rho}-\partial_{\rho} \partial_{\rho} \gamma-2\left(\partial_{z} \psi\right)^{2}+\partial_{z} \partial_{z} \psi+\frac{\partial_{\rho} \psi}{\rho}+\partial_{\rho} \partial_{\rho} \psi .
\end{aligned}
$$

Hence the vacuum field equations read

$$
\begin{gathered}
\nabla^{2} \psi \equiv \partial_{\rho} \partial_{\rho} \psi+\frac{1}{\rho} \partial_{\rho} \psi+\partial_{z} \partial_{z} \psi=0, \\
\partial_{\rho} \gamma=\rho\left[\left(\partial_{\rho} \psi\right)^{2}-\left(\partial_{z} \psi\right)^{2}\right],
\end{gathered}
$$

and

$$
\partial_{z} \gamma=2 \rho \partial_{\rho} \psi \partial_{z} \psi
$$

A very simple solution occurs when $\psi=\psi(\rho)$, then $\psi=a \ln \left(\frac{\rho}{b}\right)$ which means that the galaxy is a flat disc. Here the constant $b$ is related to the galaxy mass. Therefore a star has to obey the geodesic equations which by the line element symmetry are given by 


$$
e^{2 \psi} \dot{t}=k
$$

and

$$
\rho^{2} e^{-2 \psi} \dot{\phi}=h
$$

where $h$ and $k$ are constants which are related to energy and angular momentum. In addition the line element yields

$$
\dot{\rho}^{2}+\dot{z}^{2}=-\frac{1}{g_{11}}\left(1+g_{00} \dot{t}^{2}+g_{22} \dot{\phi}^{2}\right)
$$

where dot means derivative with relation to proper time. Performing a change of variable $u \equiv 1 / \rho$ with $z=$ constant, then

$$
\left(\frac{d u}{d \phi}\right)^{2}=-u^{2} e^{-2 \gamma}+\frac{1}{h^{2}}\left(e^{-2 \gamma-4 \psi} k^{2}-u^{2} e^{-2 \gamma-2 \psi} h^{2}\right) .
$$

Hence the constants $h$ and $k$ can be settled by

$$
\int d \phi=\int \frac{d u}{\sqrt{-u^{2} e^{-2 \gamma}+\frac{1}{h^{2}}\left(e^{-2 \gamma-4 \psi} k^{2}-u^{2} e^{-2 \gamma-2 \psi} h^{2}\right)}} .
$$

On the other hand the trajectory of the star in this model can be obtained by the following differential equation

$$
\frac{d^{2} u}{d \phi^{2}}+\left(u+\frac{d \gamma}{d \rho}\right) e^{-2 \gamma}=\frac{1}{u^{2} h^{2}}\left[\frac{d}{d \rho}(2 \psi+\gamma) k^{2} e^{-2 \psi}-\frac{d}{d \rho}(\psi+\gamma) e^{-2 \gamma-2 \psi}\right]
$$

And the tangential velocity is

$$
v_{T}=\rho \frac{\dot{\phi}}{\dot{t}}=\frac{h}{k} \frac{e^{4 \psi}}{\rho} .
$$

If $\psi$ is negative then the galactic rotation curve considering general relativity do not reproduce experimental data as can be seen from the solution for the flat disc and from the behavior of the tangential velocity against the radial distance $\rho$, in agreement with the newtonian gravitation prediction. Bičák analyzed the conditions to achieve such results [8]. This model can be improved in order to consider a fluid solution getting close to a realistic galaxy model. However we would like to consider a new variable in this scenario, i. e., the acceleration of the reference frame. Therefore in the next section the acceleration tensor have to be introduced. 


\section{ACCELERATED REFERENCE FRAMES}

In this section we briefly recall the ideas presented in [14-16]. Given a world-line $C$ the velocity field of some observer remains tangent along the curve, $u^{\alpha}=\frac{\partial x^{\alpha}}{\partial \tau}$, where $\tau$ is the proper time. Thus the velocity field is identified directly to the temporal component of the tetrad field $e_{a}{ }^{\mu}$. The tetrad field represents the components of the vector basis which has both $\mathrm{SO}(3,1)$ symmetry and diffeomorphisms. Here latin indices $a=(0)$, (i) refer to Lorentz transformations while greek ones $\mu=0, i$ to coordinate transformations. Hence the acceleration is

$$
a^{\mu}=\frac{D u^{\mu}}{d \tau}=\frac{D e_{(0)}{ }^{\mu}}{d \tau}=u^{\alpha} \nabla_{\alpha} e_{(0)}^{\mu},
$$

Then a generalization of the above expression is

$$
\frac{D e_{a}{ }^{\mu}}{d \tau}=\phi_{a}{ }^{b} e_{b}^{\mu}
$$

where $\phi_{a}{ }^{b}$ is the acceleration tensor. The absolute derivative of $e_{a}{ }^{\mu}$ establishes the kinematical state of the reference frame which is realized by the acceleration tensor. The components $\phi_{(0)}{ }^{b}$ give the translational acceleration of the observer, in order to show that, let us use 14 together with the orthogonality properties of the tetrad field, thus

$$
\phi_{a}{ }^{b}=e^{b}{ }_{\mu} \frac{D e_{a}{ }^{\mu}}{d \tau}=e^{b}{ }_{\mu} u^{\lambda} \nabla_{\lambda} e_{a}^{\mu}
$$

where $\nabla_{\lambda} e^{b}{ }_{\mu}=\partial_{\lambda} e^{b}{ }_{\mu}-{ }^{0} \Gamma_{\lambda \mu}^{\sigma} e^{b}$. Therefore

$$
a^{b}=e^{b}{ }_{\mu} a^{\mu}=e^{b}{ }_{\mu} u^{\alpha} \nabla_{\alpha} e_{(0)}^{\mu}=\phi_{(0)}^{b} .
$$

Since the tetrad field project vectors into the tangent space, a geodesic motion should be when $a^{\mu}=0$.

$$
\begin{aligned}
a^{\mu} & =u^{\alpha} \nabla_{\alpha} e_{(0)}{ }^{\mu}=u^{\alpha} \nabla_{\alpha} u^{\mu}=\frac{d x^{\alpha}}{d \tau}\left(\frac{\partial u^{\mu}}{\partial x^{\alpha}}+{ }^{0} \Gamma_{\alpha \beta}^{\mu} u^{\beta}\right) \\
& =\frac{d^{2} x^{\mu}}{d \tau^{2}}+{ }^{0} \Gamma_{\alpha \beta}^{\mu} \frac{d x^{\alpha}}{d \tau} \frac{d x^{\beta}}{d \tau} .
\end{aligned}
$$

Then as expected $\phi_{(0)}{ }^{b}$ represent the translational acceleration of the reference frame while $\phi_{(i)(j)}$ is related to rotational movement [17]. 
In riemannian geometry two connections are possible, the Christoffel symbols and the LeviCivita connection. The former one, ${ }^{0} \omega_{\mu a b}$, existis on the tangent bundle and is necessary to couple the Dirac equation to gravitational field [18]. These two quantities are related by the following identity

$$
{ }^{0} \Gamma_{\mu v}^{\lambda}=e_{a}{ }^{\lambda} \partial_{\mu} e^{a}{ }_{v}+e^{a \lambda} e^{b}{ }_{v}{ }^{0} \omega_{\mu a b}
$$

where

$$
{ }^{0} \omega_{\mu a b}=-\frac{1}{2} e^{c}{ }_{\mu}\left(\Omega_{a b c}-\Omega_{b a c}-\Omega_{c a b}\right)
$$

with

$$
\Omega_{a b c}=e_{a v}\left(e_{b}^{\mu} \partial_{\mu} e_{c}^{v}-e_{c}^{\mu} \partial_{\mu} e_{b}^{v}\right)
$$

It worths to mention that $e_{a}{ }^{\lambda} \partial_{\mu} e^{a}{ }_{\nu}$ is the Weitzenböck connection. Such a connection establishes the well known teleparallel gravity which is equivalent to general relativity. By the way $\Omega_{a b c}$ is the equivalent to the torsion tensor of Weitzenböck connection.

If expression (18) is used in (15), then the acceleration tensor simplifies to

$$
\phi_{a}{ }^{b}=-e_{(0)}{ }^{\mu} 0 \omega_{\mu}^{b}{ }_{a}
$$

which yields

$$
\phi_{a b}=\frac{1}{2}\left[\Omega_{(0) a b}+\Omega_{a(0) b}-\Omega_{b(0) a}\right] .
$$

This is the acceleration tensor in therms of the anholonomicity objects, this same expression was obtained in reference [19]. It should be noted that such a acceleration tensor does not establishes a geodesic motion, it rather describes an accelerated reference frame. 


\section{ROTATION CURVES OF GALAXIES}

A tetrad field constructed out from the line element (1) and adapted to an accelerated observer is

$$
e_{a \mu}=\left(\begin{array}{cccc}
-1 & 0 & \rho \beta(\rho, z) A(\rho, z) & 0 \\
A(\rho, z) \sin (\phi) & \alpha(\rho, z) \cos (\phi) & -\rho \beta(\rho, z) \sin (\phi) & 0 \\
-A(\rho, z) \cos (\phi) & \alpha(\rho, z) \sin (\phi) & \rho \beta(\rho, z) \cos (\phi) & 0 \\
0 & 0 & 0 & \alpha(\rho, z)
\end{array}\right),
$$

where

$$
\begin{gathered}
A^{2}-1=g_{00}=-e^{2 \psi}, \\
\alpha^{2}=g_{11}=g_{33}=e^{-2(\psi-\gamma),} \\
\beta^{2}\left(1-A^{2}\right)=e^{-2 \psi} .
\end{gathered}
$$

The inverse tetrad field reads

$$
e^{a \mu}=\left(\begin{array}{cccc}
\frac{1}{A^{2}-1} & 0 & \frac{A}{\rho\left(A^{2}-1\right) \beta} & 0 \\
\frac{A \sin (\phi)}{A^{2}-1} & \frac{\cos (\phi)}{\alpha} & \frac{\sin (\phi)}{\rho\left(A^{2}-1\right) \beta} & 0 \\
-\frac{A \cos (\phi)}{A^{2}-1} & \frac{\sin (\phi)}{\alpha} & \frac{\cos (\phi)}{\rho \beta-\rho A^{2} \beta} & 0 \\
0 & 0 & 0 & \frac{1}{\alpha}
\end{array}\right),
$$

then the 4-velocity is

$$
u^{\mu}=e_{(0)}^{\mu}=\left(-\frac{1}{A^{2}-1}, 0,-\frac{A}{\rho \beta\left(A^{2}-1\right)}, 0\right)
$$

Hence there is a rotation movement around z-axis.

The acceleration tensor reveals the kinematical state of this reference frame. Using (22) its components are given by 


$$
\begin{aligned}
& \phi_{(0)(1)}=-\frac{A \cos (\phi)\left(A-A^{3}+\rho \partial_{\rho} A+\rho A^{2} \partial_{\rho} A\right)}{\rho\left(-1+A^{2}\right)^{2} \alpha}, \\
& \phi_{(0)(2)}=-\frac{A \sin (\phi)\left(A-A^{3}+\rho \partial_{\rho} A+\rho A^{2} \partial_{\rho} A\right)}{\rho\left(-1+A^{2}\right)^{2} \alpha}, \\
& \phi_{(0)(3)}=-\frac{A\left(1+A^{2}\right) \partial_{z} A}{\left(-1+A(\rho, z)^{2}\right)^{2} \alpha}, \\
& \frac{\phi_{(1)(2)}}{A}=\frac{\left(\sqrt{\left(-1+A^{2}\right)^{2}}-A^{2}\left(\sqrt{\left(-1+A^{2}\right)^{2}}-3 \alpha\right)-\alpha-3 A^{4} \alpha+A^{6} \alpha+2 \rho A \sqrt{\left(-1+A^{2}\right)^{2}} \partial_{\rho} A\right)}{\phi_{(1)(3)}}=\frac{2 A^{2} \sin (\phi) \partial_{z} A}{\left(-1+A^{2}\right)^{2} \alpha}, \\
& \phi_{(2)(3)}=-\frac{2 A^{2} \cos (\phi) \partial_{z} A}{\left(-1+A^{2}\right)^{2} \alpha} .
\end{aligned}
$$

Then using

$$
\hat{\rho}=\cos \phi \hat{x}+\sin \phi \hat{y}
$$

and

$$
\hat{\phi}=-\sin \phi \hat{x}+\cos \phi \hat{y}
$$

we arrive at the following acceleration of the reference frame

$$
\vec{a}=-\frac{A\left(A-A^{3}+\rho \partial_{\rho} A+\rho A^{2} \partial_{\rho} A\right)}{\rho\left(-1+A^{2}\right)^{2} \alpha} \hat{\rho}-\frac{A\left(1+A^{2}\right) \partial_{z} A}{\left(-1+A^{2}\right)^{2} \alpha} \hat{z} .
$$

It should be noted that in the case of a flat disc galaxy there is no acceleration in the axial direction. Therefore the acceleration in the $\rho$-direction can be used to obtain the rotation curve for this galaxy model. Since we are dealing with a flat disc model then the potentials are

$$
\begin{aligned}
& \psi(\rho)=-X \log \left(\frac{\rho}{M}\right) \\
& \gamma(\rho)=Y \log \left(\frac{\rho}{M}\right),
\end{aligned}
$$

with $X$ and $Y$ defined as positive constants in order to yield a proper behavior for the rotation curve. Hence the acceleration is given by 


$$
a_{\rho}=\frac{\left(-1+\left(\frac{\rho}{M}\right)^{2 X}+X\left(-1+2\left(\frac{\rho}{M}\right)^{2 X}\right)\right)}{\rho\left(\frac{\rho}{M}\right)^{(X+Y)}}
$$

and the tangential velocity reads

$$
v_{T}=\sqrt{\frac{\left(-1+\left(\frac{\rho}{M}\right)^{2 X}+X\left(-1+2\left(\frac{\rho}{M}\right)^{2 X}\right)\right)}{\left(\frac{\rho}{M}\right)^{(X+Y)}}} .
$$

If $X=Y$, then it simplifies to

$$
v_{T}=\sqrt{-(M / \rho)^{2 X}+1+X\left[2-(M / \rho)^{2 X}\right]} .
$$

Particularly if $X=1$ then

$$
v_{T}=\sqrt{3-2\left(\frac{M}{\rho}\right)^{2}}
$$

which has a well defined limit for $\frac{M}{\rho}<<1$, id est, $v_{T}=$ constant. Thus the effect of an accelerated referential frame can be easily seen. It worths to point out that such a finite limit is not predicted by the newtonian theory. On the other hand it is a well known experimental data that supports dark matter. In figures 1 and 2 we plot the rotation curve considering $M$ as the galaxy mass.

It should be noted that these rotation curves are displayed in geometric unities and it remains growing until a certain limit and reaches a finite value for great distances from the galaxy center. Hence experimental data of the rotation curves can be explained as an effect of accelerated reference frame.

\section{CONCLUSION}

In this article we consider that the rotation curve of galaxies can be an effect of accelerated reference frame. We show that for a simple model of a flat galaxy the velocity of a star decreases when its distance is too far away from the galaxy center, but it remains finite. In such a model the star is supposed to describe a geodesic motion. Then an accelerated reference frame was settled by a tetrad field. The acceleration was used to obtain a galactic rotation curve and an increasing behavior was found out which is compatible with experimental data. Now other experimental 


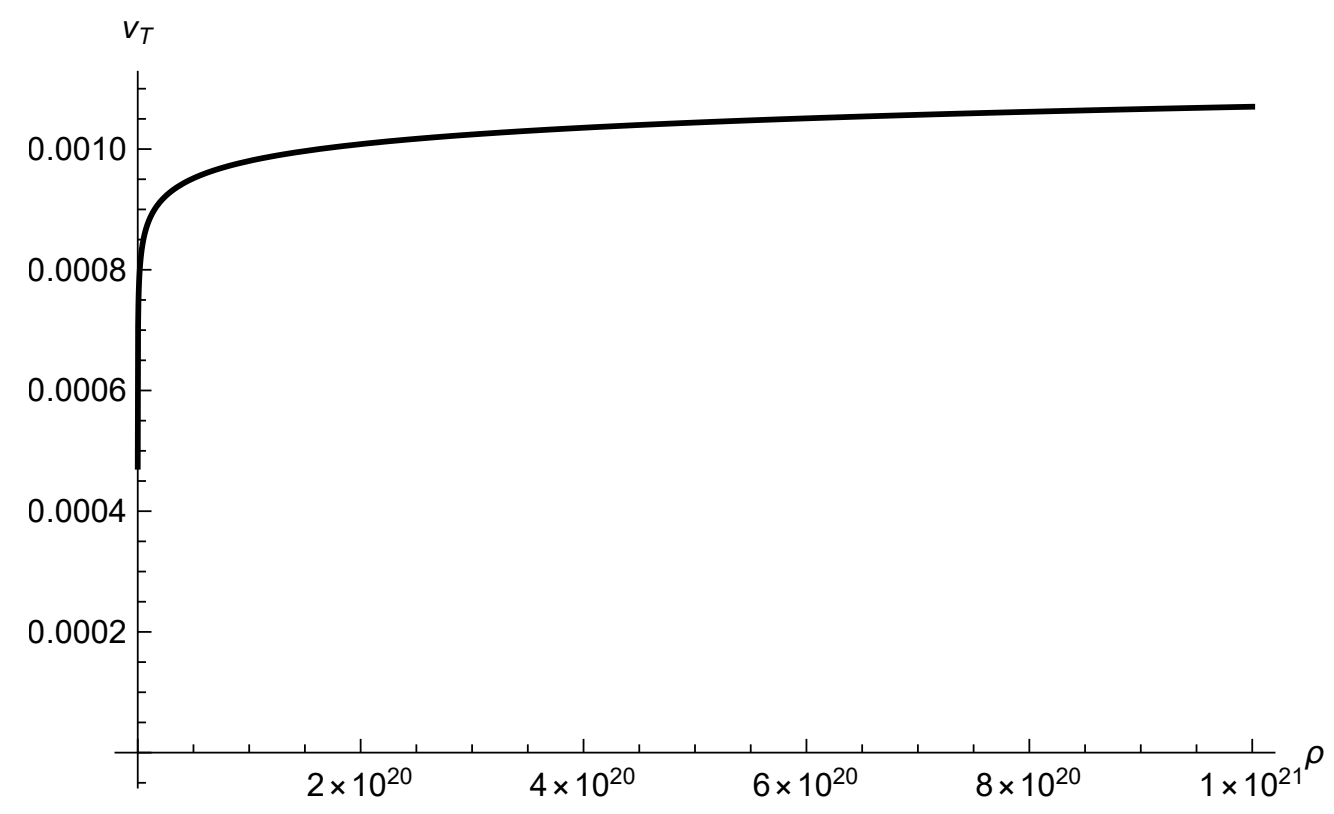

FIG. 1: Rotation curve of tangential velocity versus the distance $\rho$ for $M=10^{15} \frac{c^{2}}{G} K g$ which is suitable to describe galaxies like NGC 4594, considering $Y=X=4 \cdot 10^{-8}$.

evidences for dark matter should be confronted to our hypothesis of non inertial observer.

[1] Fritz Zwicky. Die rotverschiebung von extragalaktischen nebeln. Helvetica Physica Acta, 6:110-127, 1933.

[2] Teresa Marrodan Undagoitia and Ludwig Rauch. Dark matter direct-detection experiments. Journal of Physics G: Nuclear and Particle Physics, 43(1):013001, 2015.

[3] Timothy J Sumner. Experimental searches for dark matter. Living Reviews in Relativity, 5(1):4, 2002.

[4] Jianglai Liu, Xun Chen, and Xiangdong Ji. Current status of direct dark matter detection experiments. Nature Phys., 13(3):212-216, 2017.

[5] Mordehai Milgrom. A modification of the newtonian dynamics as a possible alternative to the hidden mass hypothesis. The Astrophysical Journal, 270:365-370, 1983.

[6] Riccardo Scarpa. Modified newtonian dynamics, an introductory review. In AIP Conference Proceedings, volume 822, pages 253-265. AIP, 2006.

[7] G. N. Candlish, R. Smith, Y. Jaffé, and A. Cortesi. Consequences of the external field effect for MOND disk galaxies in galaxy clusters. Monthly Notices of the Royal Astronomical Society, August 2018.

[8] J Bicák, D Lynden-Bell, and C Pichon. Relativistic discs and flat galaxy models. Monthly Notices of the Royal Astronomical Society, 265(1):126-144, 1993.

[9] Kenath Arun, SB Gudennavar, and C Sivaram. Dark matter, dark energy, and alternate models: A review. Advances in Space Research, 60(1):166-186, 2017. 


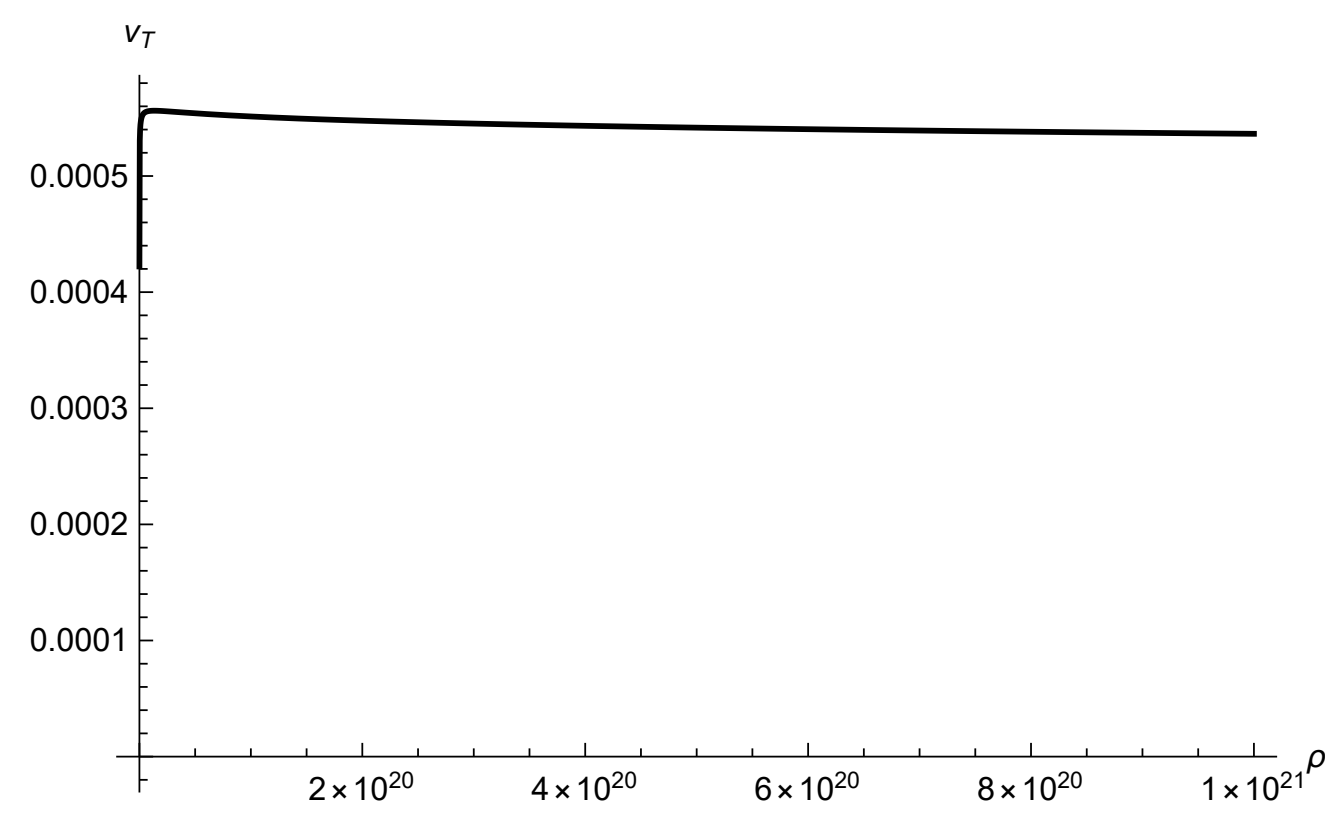

FIG. 2: Rotation curve of tangential velocity versus the distance $\rho$ for $M=10^{15} \frac{c^{2}}{G} K g$ which is suitable to describe galaxies like NGC 4594, considering $X=4 \cdot 10^{-8}$ and $Y=10^{-1}$.

[10] JW Maluf and SC Ulhoa. Electrodynamics in accelerated frames revisited. Annalen der Physik, 522(10):766-775, 2010.

[11] Sergio C Ulhoa. On dark energy and accelerated reference frames. Annalen der Physik, 524(5):273-278, 2012.

[12] PC Waylen. The general axially symmetric static solution of einsteins vacuum field equations. Proc. R. Soc. Lond. A, 382(1783):467-470, 1982.

[13] WB Bonnor. Physical interpretation of vacuum solutions of einstein's equations. part i. timeindependent solutions. General relativity and Gravitation, 24(5):551-574, 1992.

[14] Bahram Mashhoon. Nonlocality of accelerated systems. International Journal of Modern Physics D, 14(01):171-179, 2005.

[15] Bahram Mashhoon and Uwe Muench. Length measurement in accelerated systems. Annalen der Physik, 11(7):532-547, 2002.

[16] Bahram Mashhoon. Vacuum electrodynamics of accelerated systems: Nonlocal maxwell's equations. Annalen der Physik, 12(10):586-598, 2003.

[17] JW Maluf and FF Faria. On the construction of fermi-walker transported frames. Annalen der Physik, 17(5):326-335, 2008.

[18] JW Maluf. Dirac spinor fields in the teleparallel gravity: comment on metric-affine approach to teleparallel gravity. Physical Review D, 67(10):108501, 2003.

[19] JW Maluf, FF Faria, and SC Ulhoa. On reference frames in spacetime and gravitational energy in freely falling frames. Classical and Quantum Gravity, 24(10):2743, 2007. 